\title{
ICESsuHN105, a Novel Multiple Antibiotic Resistant ICE in Streptococcus suis Serotype 5 Strain HN105
}

\author{
Yinchu Zhu'1,2,3, Yue Zhang 1,2,3, Jiale Ma1,2,3, Wenyang Dong1,2,3, Xiaojun Zhong ${ }^{1,2,3}$, \\ Zihao Pan ${ }^{1,2,3}$ and Huochun Yao ${ }^{1,2,3 *}$ \\ ${ }^{1}$ College of Veterinary Medicine, Nanjing Agricultural University, Nanjing, China, ${ }^{2}$ OIE Reference Lab for Swine \\ Streptococcosis, Nanjing, China, ${ }^{3}$ Key Laboratory of Animal Bacteriology, Ministry of Agriculture, Nanjing Agricultural \\ University, Nanjing, China
}

OPEN ACCESS

Edited by: Satoru Suzuki,

Ehime University, Japan

Reviewed by:

Masatoshi Okura

National Institute of Animal Health

(NARO), Japan

William Michael McShan, University of Oklahoma Health Sciences Center, United States

${ }^{*}$ Correspondence: Huochun Yao yaohch@njau.edu.cn

Specialty section: This article was submitted to Antimicrobials, Resistance

and Chemotherapy,

a section of the journal

Frontiers in Microbiology

Received: 08 October 2018 Accepted: 01 February 2019 Published: 26 February 2019

Citation: Zhu Y, Zhang Y, Ma J, Dong W, Zhong $X$, Pan Z and Yao H (2019) ICESsuHN105, a Novel Multiple Antibiotic Resistant ICE in Streptococcus suis Serotype 5

Strain HN105.

Front. Microbiol. 10:274. doi: 10.3389/fmicb.2019.00274
Streptococcus suis serotype 5, an emerging zoonosis bacterial pathogen, has been isolated from infections in both pigs and humans. In this study, we sequenced the first complete genome of a virulent, multidrug-resistant SS5 strain HN105. The strain HN105 displayed enhanced pathogenicity in zebrafish and BABL/c mouse infection models. Comparative genome analysis identified a novel $80 \mathrm{~K}$ integrative conjugative element (ICE), ICESsuHN105, as required for the multidrug resistance phenotype. Six corresponding antibiotic resistance genes in this ICE were identified, namely tet $(\mathrm{O})$, tet (M), erm (two copies), aph, and spc. Phylogenetic analysis classified the element as a homolog of the ICESa2603 family, containing the typical family backbone and insertion DNA. DNA hybrids mediated by natural transformation between HN105 and ZY05719 verified the antibiotic resistant genes of ICESsuHN105 that could be transferred successfully, while they were dispersedly inserted with a single gene in different genomic locations of ZY05719 ${ }_{(\mathrm{HN} 105)}$ transformants. To further identify the horizontal transfer of ICESsuHN105 as a whole mobile genetic element, a circular intermediate form of ICESsuHN105 was detected by PCR. However, the effective conjugation using serotype $2 \mathrm{~S}$. suis as recipients was not observed in current assays in vitro. Further studies confirmed the presence of the complete lantibiotic locus encoded in ICESsuHN105 that effectively inhibits the growth of other streptococci. In summary, this study demonstrated the presence of antibiotic resistance genes in ICE that are able to transfer between different clinical isolates and adapt to a broader range of Streptococcus serotype or species.

Keywords: S. suis serotype 5 , comparative genomics, multi-antibiotic resistance, integrative and conjugative element, natural transformation, bacteriocin

\section{INTRODUCTION}

Streptococcus suis is a major swine pathogen that causes severe diseases in pigs, inducing meningitis, septicemia, arthritis, and endocarditis (Gottschalk et al., 2010; Fulde and Valentin-Weigand, 2013). Furthermore, S. suis is a zoonotic human pathogen affecting those in close contact with infected pigs or pig products (Huong et al., 2014; Kerdsin et al., 2017). Several outbreaks in Southeast Asia highlighted its danger to public health (Yu et al., 2006). S. suis serotype 2 (SS2) is the most 
prevalent in human infections and is relatively well studied. However, little is known about other serotypes, such as S. suis serotype 5 (SS5), which have arisen over recent decades. There have been four reported cases of human SS5 infections since 2011 around the world, including a patient with spontaneous bacterial peritonitis in Thailand, a pig farmer with septic arthritis in Sweden, a pig farmer in United States with Streptococcal toxic shock syndrome and a patient with bacteremia in Japan (Kerdsin et al., 2011; Gomez et al., 2014; Gustavsson and Rasmussen, 2014; Yoshida et al., 2017). Despite the low number of reported human cases, studying the occurrence and transmission of SS5 is important in light of the severity of the infections and the danger of the rise in antibiotic resistance. The abuse of antibiotics in multiple fields, including aquaculture and the food industry, and the genetic variation of $S$. suis via acquiring and transferring of genes, has resulted in an unprecedented rise of the antibiotic resistance phenomenon (Soares et al., 2014). Thus, it is critically important to better understand the characteristics of S. suis from both pathogenic and antibiotic-resistance perspectives.

The two significant streptococcal horizontal gene transfer mechanisms are mobile genetic elements such as integrative and conjugative elements (ICEs), and the naturally occurring competence for DNA transformation. These properties enhance bacterial adaptation to environmental conditions promoting bacterial evolution due to the acquisition of virulence factors, antibiotic resistance genes, and/or toxin-antitoxin systems (Huang J. et al., 2016). ICEs carry genes required for various survival traits, are integrative into the host chromosomes for stable transmission, and are capable of excision, circularization, and transfer via conjugation. The natural transformation abilities could mediate genomic hybridization via homologous recombination, following uptake of foreign DNA from the environment.

At the time of this study, most of the complete genomes of S. suis available in the NCBI database were SS2 strains. In this study, we present a first complete genome sequence of a virulent, multidrug-resistant SS5 (genotype) strain, HN105. A comprehensive genomic analysis with two SS2 virulent strains was performed to characterize the HN105 genome. The result demonstrated that strain HN105 contains a novel $\mathrm{ICE}(80 \mathrm{~K})$, denoted ICESsuHN105. Furthermore, the deletion of this $80 \mathrm{~K}$ genomic island confirmed the role that this ICE is required for multi-antibiotic resistance. In this study we also proved that the natural transformation for gene hybrids may show one way of horizontal gene transfer for antibiotic resistance genes between different serotype strains. Indeed, a lantibiotic gene cluster was identified in the ICE and was confirmed to confer to the donor strain a significant growth advantage in interbacterial competition.

\section{MATERIALS AND METHODS}

\section{Bacterial Strains, Culture Conditions, and DNA Extraction}

The bacterial strains used in this study are listed in Table 1. The strain HN105 was isolated from the knee joint fluid of a diseased pig in Henan province, China in 2014. The SS2 virulent strain ZY05719 was isolated in 2005 from a pig with acute septicemia during an epidemic in Ziyang, China (Zhang and $\mathrm{Lu}, 2007$ ). The reference virulent strain P1/7 was originally isolated from a diseased pig with meningitis (Wu et al., 2014b). All strains were grown in Todd-Hewitt broth (THB, BD) or on agar at $37^{\circ} \mathrm{C}$ with $5 \% \mathrm{CO}_{2}$. Chloramphenicol $(5 \mu \mathrm{g} / \mathrm{ml})$ was used, when necessary. Total genomic DNA was extracted using an Omega Bacteria DNA Kit (OMEGA, China) according to the manufacturer's instructions. All primers used in this study are listed in Supplementary Table S1.

\section{Virulence Assessment Using Zebrafish and Mouse Infection Models}

Zebrafish and BABL/c mice infections were performed according to previous studies (Wu et al., 2014a; Dong et al., 2015). The S. suis strains were grown to mid-log phase (about $\mathrm{OD}_{600}=0.6$ ), washed three times with $1 \times$ PBS $(\mathrm{pH} 7.4)$, and diluted to different doses in PBS before injection. Eight BALB/c (5week-old) mice or 15 zebrafish for each infection group were injected intraperitoneally with the bacterial suspensions and mortality was recorded for 7 days post-infection. The mice were injected with $5 \times 10^{8} \mathrm{CFU}$ and $2 \times 10^{8} \mathrm{CFU}$, then monitored three times a day; loss of mobility was set as a predictor of death during observation. Zebrafish were injected with $1 \times 10^{5} \mathrm{CFU}, 1 \times 10^{6} \mathrm{CFU}, 1 \times 10^{7} \mathrm{CFU}$, and $1 \times 10^{8} \mathrm{CFU}$. The moribund mice, recovered mice, and the mice in the control group were anesthetized at the end of the 7-day observation period with pelltobarbitalum natricum $(30 \mathrm{mg} / \mathrm{kg})$, and sacrificed by cervical dislocation. The zebrafish received lethal anesthesia with 3-aminobenzoic acid ethyl ester methanesulfonate MS-222 (90 mg/L).

\section{Growth Curve of S. suis Strains}

The overnight $S$. suis cultures in THB medium, grown to an $\mathrm{OD}_{600}$ of 0.6 , were sub-cultured again into $50 \mathrm{ml}$ THB at a 1:100 dilution. The optical densities at $\mathrm{OD}_{600}$ of bacterial cultures were measured periodically until the late stationary phase $(15 \mathrm{~h}$ after seeding, $1 \mathrm{~h}$ intervals).

\section{Antibiotic Susceptibility Test}

An antibiotic susceptibility test was performed by assessing the minimum inhibitory concentration (MIC) of strain HN105, P1/7 and ZY05719. Eighteen antibiotics were used, including some important antibiotics such as erythromycin, vancomycin, tetracycline, cephalosporins, chloramphenicol and kanamycin. The test was performed by the broth microdilution method, according to the standard methods in the Clinical and Laboratory Standards Institute (CLSI) guidelines (2016). Briefly, the overnight bacterial culture was diluted (1:1000). Equal volumes of the bacterial culture were added in the 96-well plates and mixed with serially diluted antibiotics. Following $16 \mathrm{~h}$ incubation at $37^{\circ} \mathrm{C}$ with $\mathrm{CO}_{2}$, the growth in each well was recorded. The highest dilution of the antibiotic that inhibited bacterial growth, compared to the media control, was recorded as the MIC. 
TABLE 1 | The information of sequenced strains used for genomic comparison.

\begin{tabular}{lcccccl}
\hline Strains & Serotype & Size(M) & GC\% & ST & $\begin{array}{c}\text { GenBank } \\
\text { accession } \\
\text { number }\end{array}$ & $\begin{array}{l}\text { Place of } \\
\text { origin }\end{array}$ \\
\hline ZY05719 & 2 & 1.99 & 41.1 & 7 & CP007497.1 & China \\
P1/7 & 2 & 1.91 & 41.2 & 1 & NC_012925.1 & Europe \\
\hline
\end{tabular}

\section{High-Throughput Genome Sequencing, Assembly, and Annotation}

The whole genome sequencing of HN105 was performed on the Illumina Miseq and Pacbio RSII platforms (Personalbio BioTechnology Co., Ltd.). Using SPAdes (version 3.7.1), and the second generation and third generation sequencing data were assembled. Using the MUMmer software for co-linearity analysis with the reference genome, the positional relationship of contigs on the genome was determined. The gaps between the contigs were repaired using the original data from the third-generation sequencing. Finally, the complete chromosomal sequence was corrected using Pilon software. For plasmids, the contigs were compared to the nucleotide library in the NCBI database, the plasmid sequence was picked out, and the third-generation sequencing data was used to fill the gaps as noted above in order to obtain a complete plasmid sequence. A total of 2,196,724 bp for chromosomal DNA were generated by the combination of the two libraries (PE400 and S10K). The open reading frames (ORFs) were identified using Glimmer 3.0 based on the HN105 genome sequence (Delcher et al., 1999). The tRNAs and rRNAs were predicted by tRNAscan-SE 1.31 and RNAmmer 1.2, respectively (Lowe and Eddy, 1997), whereas ncRNA was BLAST-analyzed to the Rfam database (Lagesen et al., 2007). The CRISPR recognition tool (CRT) was used to predict DRs (forward repeats) and spacers (Bland et al., 2007). The GIs of HN105 were determined with Island Viewer ${ }^{1}$. The sequences and annotations of HN105 were deposited with GenBank ${ }^{2}$ under accession no. CP029398 and no. CP029399 for the plasmid.

\section{Multi-Locus Sequence Typing (MLST)}

By comparing the sequences of seven housekeeping genes (aroA, cpn60, dpr, gki, mutS, recA, and thrA) in S. suis, MLST provides a discriminatory genotyping method for different strains (Maiden et al., 2013). The allele numbers and sequence type (ST) of strains used in this study were downloaded from the MLST database ${ }^{3}$ and analyzed with eBURST ${ }^{4}$.

\section{Comparative Genomic Analyses of HN105}

Strains P1/7 and ZY05719 were representative clinical and virulent SS2 strains, respectively (Table 1). A comparison of HN105 with the above SS2 strains were performed using the

${ }^{1}$ http://www.pathogenomics.sfu.ca/islandviewer/upload/

${ }^{2}$ http://www.ncbi.nlm.nih.gov/genbank/

${ }^{3}$ http://ssuis.mlst.net/

${ }^{4}$ http://eburst.mlst.net/ program progressiveMauve (Darling et al., 2010), displaying multiple genome alignments.

\section{Determination of the ICESsuHN105 Sequence}

A comparison of HN105 with P1/7 and ZY05719 was performed using progressiveMauve (Darling et al., 2010) and a major genomic island discovered was determined to be an ICE element.

Annotation of the complete ICE sequence was conducted with RAST (Aziz et al., 2008) and the results were manually checked by comparison with the NCBI database ${ }^{5}$. Comparative analysis was carried out with the BLAST program ${ }^{6}$.

\section{Phylogenetic Analysis of the ICEs in S. suis}

A recent study identified 30 core genes of ICEs in S. suis, primarily those encoding integrase, excisionase, relaxase, plasmid mobilization relaxosome protein, type IV secretion systems, DNA primase, replication initiator protein A, DNA 5'methyltransferase and hypothetical proteins (Huang J. et al., 2016). Using this data, we compared the HN105 ICE genes with the corresponding genes of ICESa2603 using BLAST analysis and the ACT software in order to build phylogenetic trees. In all, 13 ICEs were used to generate the phylogenetic tree on MEGA6, using the maximum-likelihood method with bootstrapping.

\section{Construction of the $\Delta 80 \mathrm{~K}$ Mutant and Its Role in Antibiotic Resistance}

The unique $80 \mathrm{~K}$ genomic island was knocked out by natural transformation, according to a recent study (Zaccaria et al., 2014). The forward and reverse homologous sequences of the target gene were fused with the chloramphenicol marker by overlap PCR. Then the DNA products were mixed with the peptide, incubated, and selected on THB agar $\left(\mathrm{Cm}^{\mathrm{R}}\right)$. MICs were then determined for kanamycin, gentamicin, erythromycin, spectinomycin, tetracycline, lincomycin, and neomycin.

\section{Construction of ZY05719 Multi-Antibiotic Transformants}

Genomic DNA was extracted from HN105 using the Omega genomic DNA purification kit (OMEGA, China). The transformants were generated by natural transformation of HN105 DNA into ZY05719 (SS2) on THB agar, as described previously (Zaccaria et al., 2014). The receptor strain ZY05719 was cultured in THB until OD about 0.04, and HN105 DNA with pheromones peptide were mixed with $100 \mu \mathrm{THY}$ bacteria. After $2 \mathrm{~h}$ of incubation at $37^{\circ} \mathrm{C}$, samples were spread on plates $\left(\mathrm{Kan}^{+}\right.$and $\left.\mathrm{Spc}^{+}\right)$. Of note, HN105 is multidrug-resistant (spectinomycin, kanamycin, tetracycline and erythromycin) and conferred chromosomally, whereas ZY05719 is susceptible to all three. The antibiotic resistance of transformants was tested on THB agar with $100 \mu \mathrm{g} / \mathrm{ml}$ spectinomycin and $50 \mu \mathrm{g} / \mathrm{ml}$

\footnotetext{
${ }^{5}$ https://www.ncbi.nlm.nih.gov/

${ }^{6}$ http://blast.ncbi.nlm.nih.gov/Blast.cgi
} 
kanamycin. The transformants were cultured and the DNA extracted for genome sequencing.

\section{Detection of Excision and Circularization of ICESsuHN105, and Mating Assays}

The integrated form and extrachromosomal circular form of the ICEs in S. suis were detected by combination primers, P1-P4. The amplicons were sequenced by Sanger sequencing and aligned with the attL and attR of ICESsuHN105.

For mating experiments, the mutant strain P1/7 $\left(\mathrm{Cm}^{\mathrm{R}}, \mathrm{Tet}^{\mathrm{S}}\right.$, and $\mathrm{Ery}^{\mathrm{S}}$ ) was used as a recipient and the multi-drug resistant strain HN105 as the donor. The filter mating assays were performed as previously described (Huang J. et al., 2016;Huang K. et al., 2016). The cultured donor and recipient strains, at an $\mathrm{OD}_{600}$ of $0.4-0.6$, were mixed at a ratio of $1: 5$. The cell mixtures were resuspended, spotted on filter membranes, and were grown on THB agar plates overnight. The transconjugants were selected using the antibiotics tetracycline/erythromycin and chloramphenicol. Finally, the transconjugants were further confirmed by PCR for the ICESsuHN105 genes $r e c N, g d h$, and $c p s 2$.

\section{Construction of $\Delta$ Sss Mutant and Antimicrobial Susceptibility Test}

The lantibiotic cluster deletion mutant strain $\Delta S s s\left(\mathrm{Cm}^{\mathrm{R}}\right)$ was constructed as described above. For antimicrobial susceptibility testing, $5 \mu \mathrm{L}$ of $\mathrm{OD}_{600} 0.6$ Streptococcus culture was placed on the surface of THB agar $\left(1.5 \%\right.$, w/v) plates and incubated at $37^{\circ} \mathrm{C}$ under $5 \% \mathrm{CO}_{2}$ for $24 \mathrm{~h}$. Melting agar (0.5\% agar, w/v) medium was prepared and mixed with the indicator bacteria at about $50^{\circ} \mathrm{C}$. The mixing agar medium $(5 \mathrm{~mL})$ containing $10^{6} \mathrm{CFU}$ indicator bacteria was overlaid on the plate uniformly and then incubated at $37^{\circ} \mathrm{C}$ for $24 \mathrm{~h}$. The antimicrobial susceptibility was measured by the appearance and diameter of the inhibition zone. The lantibiotic extraction was performed as previously described (Vaillancourt et al., 2015) and details are provided in the Supplementary Material.

\section{RESULTS}

\section{Characteristics of the Strain HN105}

The bacterial strain isolated from a diseased pig with acute arthritis was identified as $S$. suis according to species-specific PCR (detecting $g d h$ and $r e c N$ ) and 16S rRNA sequencing (Ishida et al., 2014), and named HN105. A remarkable self-agglutination resulted in a negative serologic agglutination test. Serotypespecific PCR identified HN105 as a type 5 strain of $S$. suis in genotype and showed $\alpha$-hemolysis on $5 \%(\mathrm{v} / \mathrm{v})$ sheep blood agar (Supplementary Figure S1). As HN105 was not serologically identified in the phenotype, we marked it as SS5 (genotype) here.

The in vitro growth characteristics of $\mathrm{HN} 105$ were compared with two other S. suis strains (P1/7 and ZY05719) in THB. The results indicated that the SS5 (genotype) strain HN105 grew faster at early logarithmic phase (Supplementary Figure S2).

\section{HN105 Exhibited High Virulence in Zebrafish and BABL/c Mouse Infection Models}

To evaluate the virulence of HN105 in vivo, we first used an established zebrafish model to compare its pathogenicity with two well-known SS2 virulent strains, P1/7 and ZY05719. The LD50 values for strains HN105, P1/7 and ZY05719 were $7.65 \times 10^{5} \mathrm{CFU} /$ fish, $1.08 \times 10^{6} \mathrm{CFU} / \mathrm{fish}$, and $3.52 \times 10^{5} \mathrm{CFU} /$ fish, respectively (Figure 1A). This result implied that the virulence of strain HN105 is close to that of ZY05719 and higher than that of P1/7 in the zebrafish model (Wu et al., 2014a).

Next, we used the BABL/c mouse infection model to reassess the virulence of HN105. As shown in the survival curves (Figure 1B), the HN105 strain was more pathogenic to BABL/c
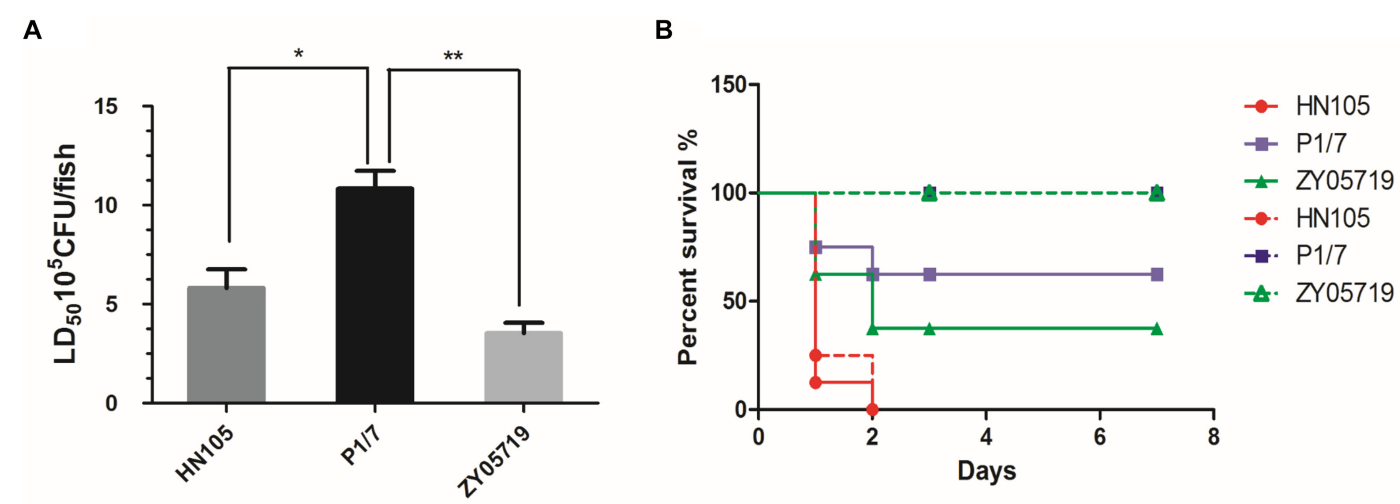

Strains

FIGURE 1 Animals experiments for strains HN105, P1/7 and ZY05719. The zebrafish (A) and BABL/c mouse (B) infection model were used to compare the virulence of SS5 (genotype) HN105 and SS2 P1/7, ZY05719. The dotted line means BABL/c mice with injection dose of $5^{*} 10^{7} \mathrm{cfu}$. The solid line means BABL/c with injection dose of $2 * 10^{8} \mathrm{cfu}$. The zebrafishes and BABL/c mice were observed 7 days after challenge. Statistical analyses were performed using one-way AVOVA and Tukey's multiple comparisons test with all columns. $P<0.05$ is labeled with *, $P<0.01$ is labeled with **. 
mice than the two SS2 strains, further supporting the idea that HN105 should be classified as part of the virulent group of S. suis (Dong et al., 2015). Furthermore, some of the mice infected with HN105 developed typical disease symptoms, including shivering and dyspnea.

\section{Antibiotic Resistance Profiles}

The results of the MIC test were tabulated (Table 2). The data demonstrated that $\mathrm{HN} 105$ was resistant to many antibiotics such as tetracycline, kanamycin, gentamicin, erythromycin, lincomycin, neomycin, and spectinomycin. The S. suis strains P1/7 and ZY05719 were susceptible to these antibiotics, except for tetracycline, gentamicin and neomycin. This multidrug resistance, coupled with the high virulence of HN105 in animal infection assays, prompted a comprehensive genomics analysis of the SS5 (genotype) strain, HN105.

\section{General Features of the HN105 Genome}

The genomic sequencing of strain HN105 revealed a 2,131,882 bp circular chromosome and a 20,752 bp plasmid (Figure 2). The general features of the genome are summarized in Supplementary Table S2. In total, 2077 coding sequences (CDSs) were identified in the chromosome and 27 coding sequences on the plasmid. The average $\mathrm{G}+\mathrm{C}$ contents were 41.4 and $33.22 \%$ for the chromosome and plasmid, respectively. Additionally, the tRNA and rRNA cluster copy numbers were similar to the other SS2 strains, and only one type of CRISPR was identified. MLST analysis identified that HN105 belongs to sequence type 498, which harbors another SS5 strain, Sdly140101.

TABLE 2 | The MIC of several antibiotics against Streptococcus suis HN105 and other two SS2 strains.

\begin{tabular}{|c|c|c|c|c|}
\hline Antibiotic & HN105 & $\mathrm{P} 1 / 7$ & ZY05719 & $\begin{array}{c}\text { Resistance } \\
\text { breakpoint }\end{array}$ \\
\hline \multicolumn{5}{|c|}{ MIC ( $\mu \mathrm{g} / \mathrm{ml})$} \\
\hline Vancomycin & 0.5 & 0.125 & 0.125 & $\geq 2$ \\
\hline Tetracycline & 128 & 32 & 32 & $\geq 8$ \\
\hline Cephalosporins & $<0.125$ & $<0.125$ & $<0.125$ & $\geq 8$ \\
\hline Chloramphenicol & 8 & 1 & 2 & $\geq 16$ \\
\hline Kanamycin & $>256$ & 8 & 16 & - \\
\hline Erythromycin & $>256$ & $<0.125$ & $<0.125$ & $\geq 1$ \\
\hline Penicillin & $<0.125$ & $<0.125$ & $<0.125$ & $\geq 8$ \\
\hline Lactobacillus & $>256$ & $>256$ & $>256$ & - \\
\hline Ampicillin & $<0.125$ & $<0.125$ & $<0.125$ & $\geq 8$ \\
\hline Streptomycin & $>256$ & 4 & $>256$ & - \\
\hline Doxycycline & 32 & 0.25 & 0.25 & $\geq 16$ \\
\hline Ciprofloxacin & 64 & 8 & 32 & $\geq 4$ \\
\hline Gentamicin & 32 & 16 & 16 & $\geq 16$ \\
\hline Amikacin & 128 & 128 & 128 & $\geq 64$ \\
\hline Norfloxacin & 64 & 64 & 64 & $\geq 32$ \\
\hline Lincomycin & $>256$ & 0.5 & 1 & $\geq 1$ \\
\hline Neomycin & $>256$ & 128 & 128 & - \\
\hline Spectinomycin & $>256$ & 8 & 32 & - \\
\hline
\end{tabular}

"-" means there is no suitable breakpoint of S. suis for the AMR from CLSI.

\section{Comparative Genomic Analysis and Identification of a Novel ICE}

Comparative genomic analysis of HN105, the reference virulent SS2 strain P1/7 and ZY05719 was performed to better understand the multidrug resistance of the SS5 (genotype) strain. Genome alignment showed large-scale genomic rearrangements in HN105, including inversions, insertions and deletions. Although there was no typical $89 \mathrm{~K}$ PAI encoded in HN105, a unique $80 \mathrm{~K}$ genomic island (GI) was seen inserted in the chromosome. This GI was 79,639 bp in size with an average GC content of $38.04 \%$, significantly different from that of the whole genome (41.4\%). Genes encoding transposase and integrase were found in its flanking region. Although a lot of genes in $80 \mathrm{~K}$ GI encode hypothetical proteins, we identified some antibiotic resistancerelated genes or regulators, which will be interesting for further analysis (Figures $\mathbf{3 A}, \mathbf{B})$. In addition, there were two another GIs (43K and $40 \mathrm{~K}$ ) inserted into the chromosome, composed primarily of accessory secretion systems, glycosyltransferase and prophage elements.

Despite the large-scale genomic differences and the absence of important SS2-associated virulence genes such as $m r p$ or sly in the chromosome of HN105, we identified some virulencerelated factors such as $f b p, \operatorname{srt} A$, dippIV, enolase, an orphan regulator, $\operatorname{cov} R$ and the $n a d R$ regulator. In addition, the capsule synthesis gene $c p s$ and the capsule regulatory genes $c c p A$ were also identified in HN105. Expanding the search to genes coding for products potentially involved in bacteremia-enhancing $S$. suis virulence revealed the presence of important virulence-related genes (Supplementary Table S3).

Further analysis of the $80 \mathrm{~K}$ genomic island confirmed that it was a novel ICE element, possessing a conserved integrase closely related to intICESa2603 (>99\% amino acid identity). Moreover, alignment of the core genes with ICESa2603 showed $>60 \%$ DNA sequence identity. Both the above indexes are specific features of the ICESa2603 family ${ }^{7}$. Accordingly, the HN105 ICE was renamed ICESsuHN105 and classified as a member of the ICESa2603 family.

\section{Phylogenetic and Neighborhood Analysis of ICESsuHN105}

In ICESsuHN105, integration between the rpsL and hydrolase genes yielded two 15 bp att sites at the left and right ends (attL/R: 5'-TTATTTAAGAGTAAC-3'). A putative oriT sequence was identified upstream of the mobC gene, at the genomic locus 1015147 to 1015183 (GGGATATTGTGGACACAATATCTGAGCTCGCAAAGAC), identical to ICESa2603. In addition to possessing genes involved in conjugation, which were annotated as a tyrosine family integrase, Tn5252 orf4 relaxase, Tn5252 orf9 mobC and Tn5252 orf10, ICESsuHN105 also contained some accessory genes conferring growth advantages (Figure 3C). Furthermore, ORFs predicted to encode the PezAT toxin-antitoxin system were verified to be functional and transcribed as an operon mode (Figure 3C).

\footnotetext{
${ }^{7}$ http://db-mml.sjtu.edu.cn/ICEberg/index.php
} 
A

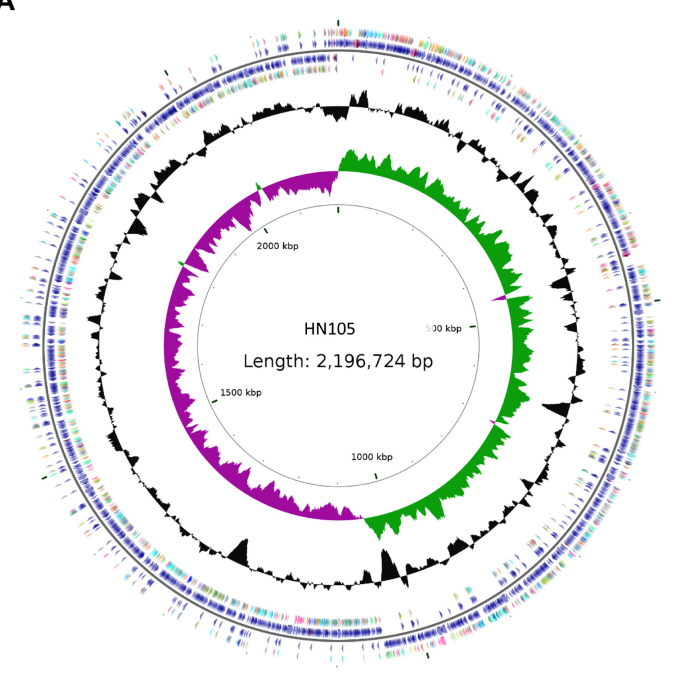

B

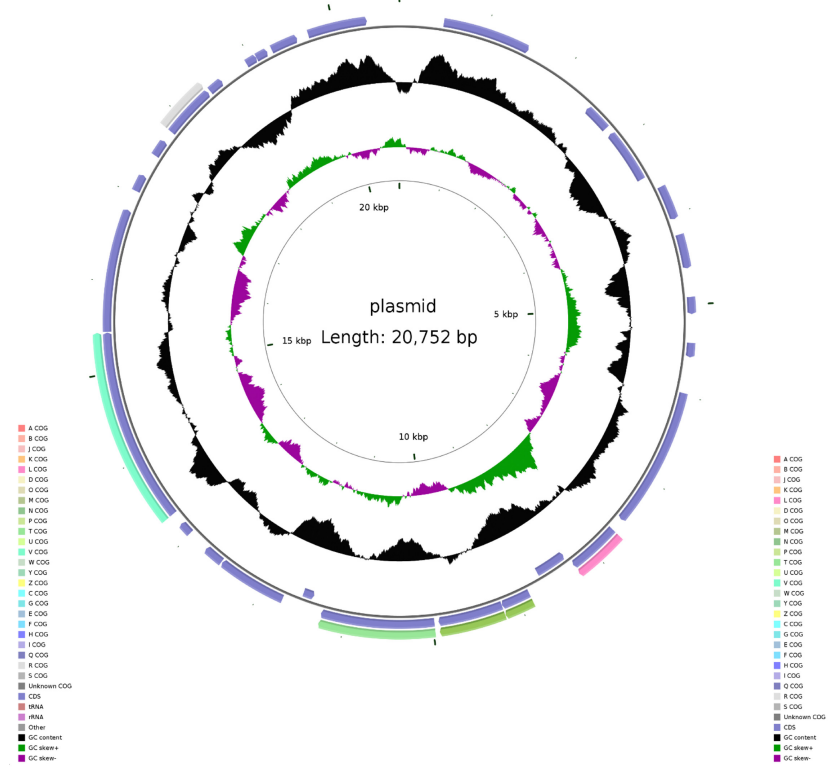

FIGURE 2 | Circle map of the genome of S. suis HN105 (A) and its plasmid (B). Map was established using the software CGView. The key information pertains to the circular diagrams (outside to inside): the outermost ring shows annotated CDSs, tRNA, rRNA, and Gls; the second ring shows GC content; the innermost ring shows $G C$ skew $[(G-C) /(G+C)]$.
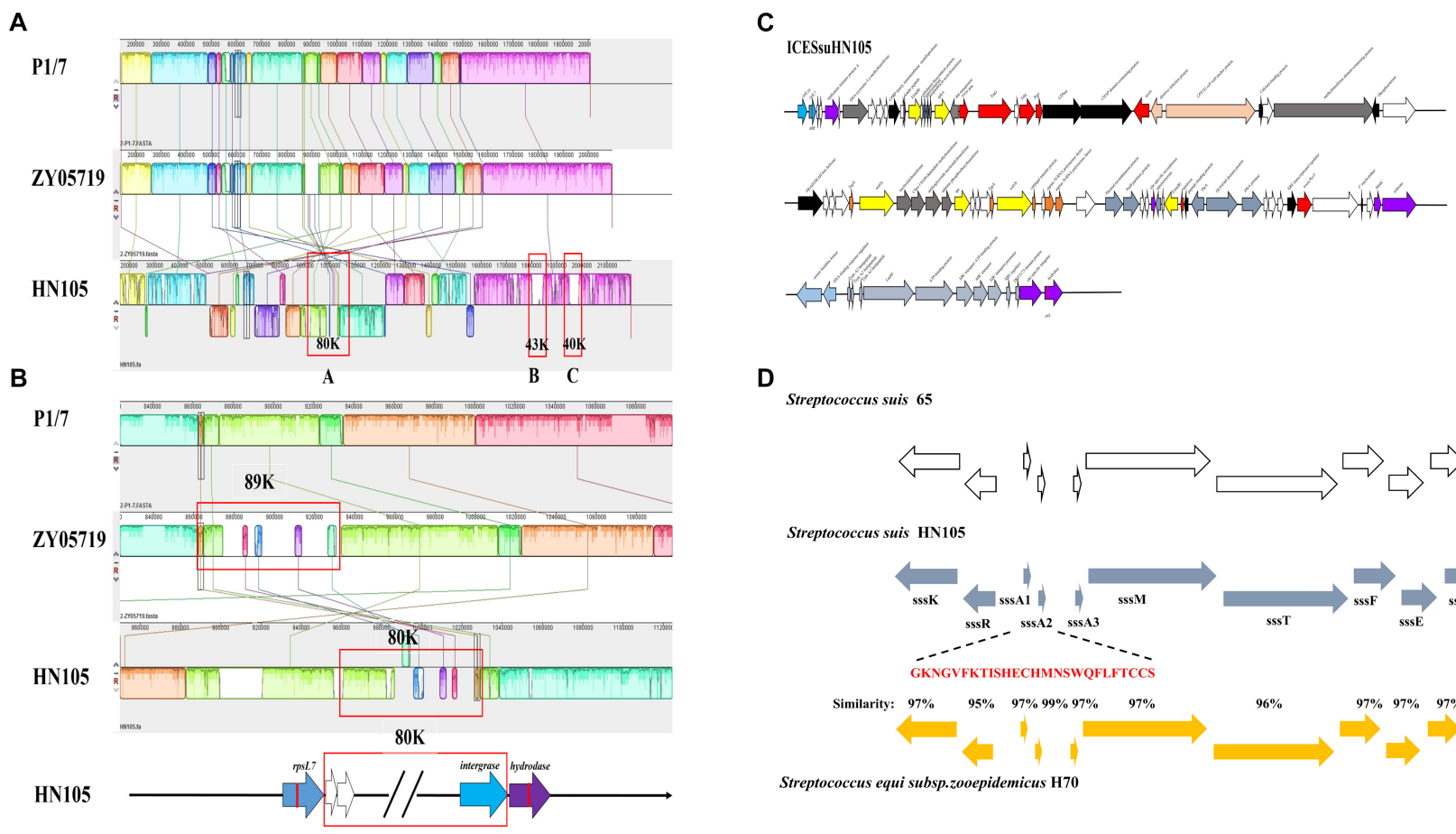

D

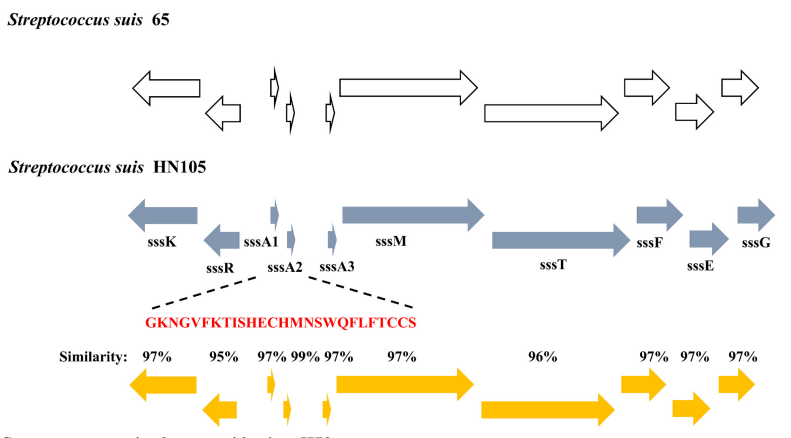

Streptococcus equi subsp.zooepidemicus $\mathrm{H70}$

FIGURE 3 | (A) Mauve comparison diagrams of the HN105, P1/7, and ZY05719 genomes. The map of an 80K genomic island, 43K genomic island and 40K genomic island were present in HN105 genome but absent in the virulent SS2 strains. (B) The enlarged map of 80K in HN105 and 89K in ZY05719. Each colored region is a locally collinear block (LCB). (C) A schematic map of the genetic structure of ICESsuHN105. The transcriptional directions and relative size of the ORFs are indicated by the arrows. The white arrows indicate unknown genes. The gray-blue arrows refer to the locus of lantibiotic cluster and the yellow arrows indicate the antibiotic resistant genes. The red arrows represent the T4SS system elements. The rest of the colored arrows are the essential genes for the complete function of ICEs. (D) An alignment between the type-B lantibiotic locus from S. suis strain 65 (white bars), S. equi subsp. H70 (yellow bars) and the corresponding locus within strain HN105 (blue bars) are shown. The nisin genes in S. suis are designated as SSA1 and SSA2. The mature polypeptide is marked in red. 


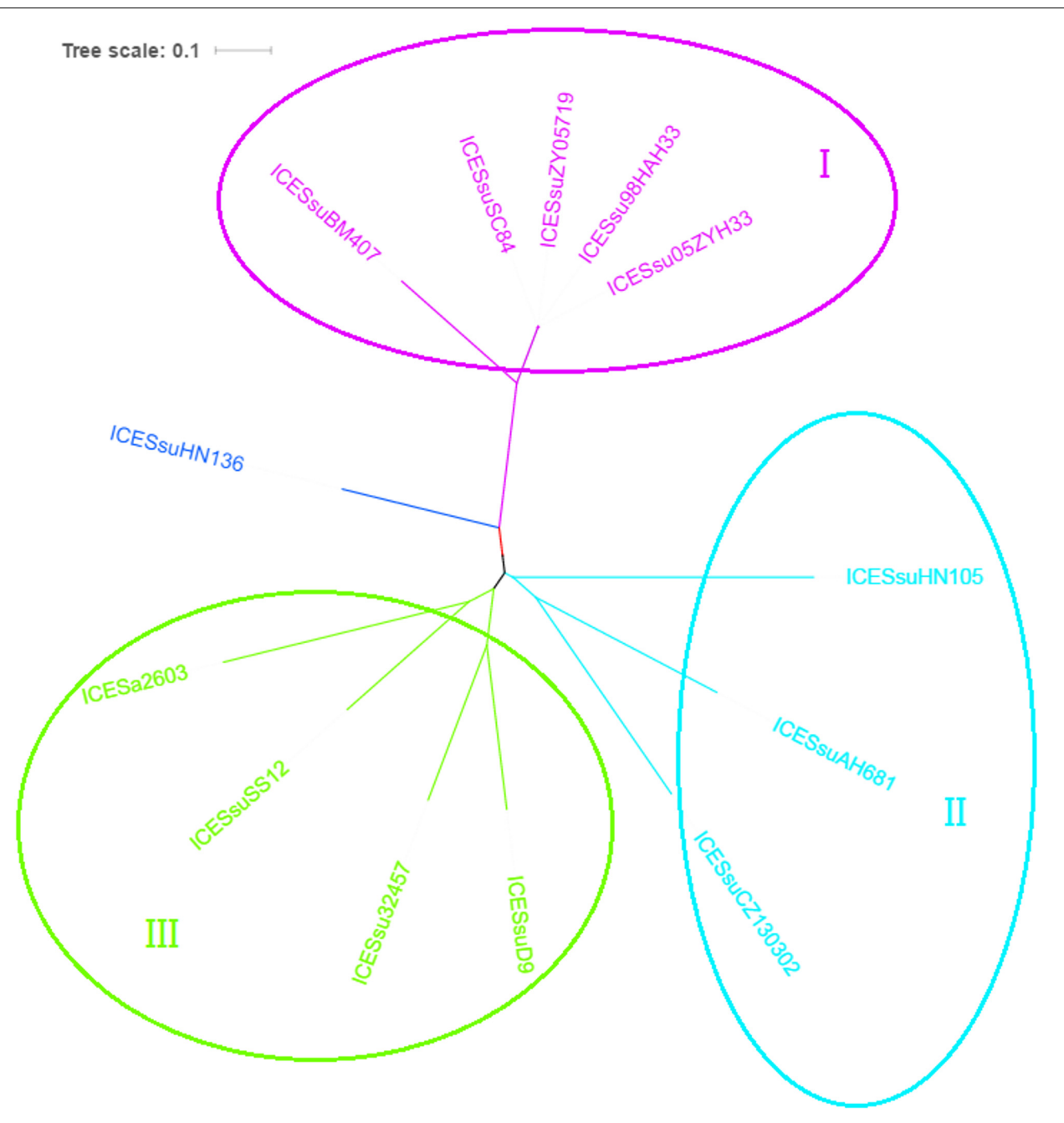

FIGURE 4 | Phylogenetic tree of ICESa2603 family in S. suis based on backbone sequences built by concatenating core genes. The tree was constructed by maximum-likelihood method using MEGA6.

As expected, the analysis results showed that ICESsuHN105 contains several antibiotic resistance-associated determinants. Examples include HN015_04855 encoding an aminoglycoside $O$-phosphotransferase (APH), which could confer resistance to multiple antibiotics including kanamycin, lincomycin, neomycin, and amikacin; two identical genes (HN015_04830 and HN015_05090) encode rRNA adenine N-6-methyltransferases for erythromycin resistance; genes HN015_05015 and HN015_04965 encode ribosomal protection proteins, which could protect the ribosome from the translation inhibition of tetracycline; and spectinomycin adenylyltransferase (HN015_04990) could result in spectinomycin resistance. Notably, a complete lantibiotic locus was encoded in this ICE (Figure 3D).

To analyze the evolution of the ICE core cluster in S. suis, we compared the DNA sequence identity of each of the ICE core genes to the corresponding genes of ICESa2603 (Figure 4). The phylogenetic trees based on the core genes demonstrated that the ICEs were further divided into three subgroups, with the divergent branch (group III) containing only one member, ICESsuHN136. Group I included 89K-like ICEs, most of them containing critical virulence-related factors, and tetracycline and macrolide resistance genes (Tang et al., 2006; Li et al., 2011). Three novel ICEs comprised group II, and included ICESsuHN105, ICESsuAH681 and ICESsuCZ130302. ICESsuCZ130302 and ICESsuAH681 were detected in novel serotype Chz strains. In addition, ICESsuCZ130302 has been demonstrated to be excisable from the donor cell chromosome, cyclized and transferred to the S. suis serotype 2 virulent reference strain P1/7 (Pan et al., 2019). These ICEs carried multiple antibiotic resistance genes instead of VFs.

\section{Effective Role of ICESsuHN105 in Resistance to Multiple Antibiotics}

The resistance profiles of the wild-type and mutant $\Delta 80 \mathrm{~K}$ strains were compared for 7 antibiotics, to which HN105 and ZY05719 had been identified to be resistant and sensitive, respectively (Table 2). We found that the mutant strain was not resistant to any of these antibiotics (Table 3). Based on the data, we 
could preliminarily conclude that ICESsuHN105 is effective in conferring antibiotic resistance to HN105.

\section{Multidrug Resistance of ZY05719 (HN105) Results From the Insertion of Resistant Genes in Different Genomic Locations}

The transmissibility of multidrug resistance from HN105 to the different S. suis strains was determined. Here we devised an experimental strategy to identify the genetic changes causing antibiotic resistance (Supplementary Figure S4). This strategy was involved to induce genomic hybrids by natural transformation between two $S$. suis strains with different phenotypic antibiotic sensitivity. Accordingly, purified HN105 genomic DNA was used as the donor DNA, with the SS2 ZY05719 being the recipient strain. The positive clones screened on THB plates containing multiple antibiotics were named ZY05719(HN105) transformants. The antibiotic resistance of $\mathrm{ZY05719}{ }_{(\mathrm{HN} 105)}$ was further investigated by MIC assays. The results indicated an increase in the MICs of spectinomycin, kanamycin, neomycin, tetracycline, and erythromycin for the ZY05719 ${ }_{(\mathrm{HN} 105)}$ transformants compared to the parental strain (Table 4). This result indicated the antibiotic resistant genes could easily be horizontally transferred between clinical isolates from different serotypes under environmental antibiotic stress.

To explore whether horizontal transfer of antibiotic genes occurred by an insertion of the whole ICESsuHN105 box in the ZY05719 genome, one ZY05719(HN105) transformant was sequenced and the draft genome was analyzed (GanBank ID: RZIC00000000). The genomic data suggested that the different antibiotic resistance elements from ICESsuHN105 were dispersedly inserted as single genes

TABLE 3 | Comparison of aminoglycosides antibiotics between S. suis HN105 and $\triangle 80 K$.

\begin{tabular}{lcc}
\hline Antibiotic & HN105 & $\mathbf{\Delta 8 0 K}$ \\
\hline & MIC $(\boldsymbol{\mu} \mathbf{g} / \mathbf{m l})$ & \\
\hline Kanamycin & 4096 & 32 \\
Gentamicin & 32 & 16 \\
Neomycin & 1024 & 128 \\
Erythromycin & 4096 & $<0.125$ \\
Tetracycline & 128 & 16 \\
Spectinomycin & 4096 & 8 \\
Lincomycin & 1024 & 0.5 \\
\hline
\end{tabular}

TABLE 4 | The MIC results of ZY05719 ${ }_{\mathrm{HN} 105}$ transformants.

\begin{tabular}{lccc}
\hline Antibiotic & HN105 & ZY05719 $_{\mathbf{H N 1 0 5}}$ & ZY05719 \\
\hline & MIC $(\boldsymbol{\mu} \mathbf{g} / \mathbf{m l})$ & & \\
\hline Kanamycin & $>256$ & $>256$ & 32 \\
Erythromycin & $>256$ & $>256$ & $<0.125$ \\
Spectinomycin & $>256$ & $>256$ & 32 \\
Tetracycline & 128 & 128 & 32 \\
Neomycin & $>256$ & $>256$ & 128
\end{tabular}

at different genomic locations. Although the acquisition of novel antibiotic resistance genes was confirmed by the contribution of the individual HN105-encoded genes, the experiments did not identify the horizontal transfer of intact ICESsuHN105 by natural transformation in Streptococcus species.

\section{Detection of Extrachromosomal Circular Forms of ICESsuHN105}

Active ICEs can be excised from the chromosome with the aid of the integrase and excisionase to form circular extrachromosomal ICEs. Four specific primers (P1, P2, P3, and P4) targeting HN105 were designed in order to detect ICESsuHN105. PCR with different combinations of the four primers can be used to identify both the chromosomally integrated (P1-P2 and $\mathrm{P} 3-\mathrm{P} 4$ positive; $\mathrm{P} 2-\mathrm{P} 3$ and $\mathrm{P} 4-\mathrm{P} 1$ negative) and excisedcircularized (P1-P2 and P3-P4 negative; P2-P3 and P4-P1 positive) forms of ICESsuHN105 in S. suis HN105 grown to logarithmic phase. The PCR analysis showed that DNA amplicons were obtained with all these four pairs of primers; however, the bands of P2-P3 and P4-P1 were shallower. The results indicated that although the probability of occurrence is relatively low, both the forms of ICESsuHN105 (chromosomally integrated, and excised-circularized) occurred when strain HN105 was cultured. This suggested that ICESsuHN105 had the ability to form an annular structure (Figure 5). However, successful conjugants between donor S. suis HN105 and recipient strains $\mathrm{P} 1 / 7\left(\mathrm{Cm}^{\mathrm{R}}\right)$ have not been obtained in our in vitro conjugation assays.

\section{Identification of a Functional Lantibiotic Locus Located in ICESsuHN105}

According to the comparative genomics analysis, there is a type B lantibiotic locus at the end of the 80K ICESsuHN105; an identical gene order to the nisin locus in $S$. equi subsp. strain H70 (Holden et al., 2009) and S. suis 65 (Vaillancourt et al., 2015). Based on the annotation, this complete lantibiotic locus (suicin locus) contains 10 coding genes, including a sensor histidine kinase (sssK), a response regulator (sssR), three precursors (sss $A 1$, $s s s A 2$, and sss 3 ), a lantibiotic modification ( $s s s M)$, an ABC transporter $(s s s T)$, and three immunity proteins $(s s s F, s s s E$, and $s s s G$ ) (Figure 3D). Therefore, we redesignated this locus as 'locus suicin 105 .'

The plate diffusion assay was used to investigate the antimicrobial ability of HN105 to other streptococcal strains. The data showed that S. agalactiae A909, S. equi subsp. zooepidemicus ATCC35246, S. suis P1/7, and S. pneumoniae RX1 were sensitive to HN105, but S. agalactiae GD1008-001 and S. suis ZY05719 were not (Figure 6A). Therefore, S. agalactiae A909 was selected as the indicator strain in further studies.

To verify that the observed antimicrobial ability of HN105 was conferred by suicin 105 , a mutant strain $\Delta$ Sss was constructed. Compared to the wild type, $\Delta$ Sss displayed significantly smaller inhibitory zones in plate diffusion assays using S. agalactiae A909 as the indicator (Figure 6B). This result indicated that suicin 105 contributes to antimicrobial activity of HN105 against other streptococcal strains. 


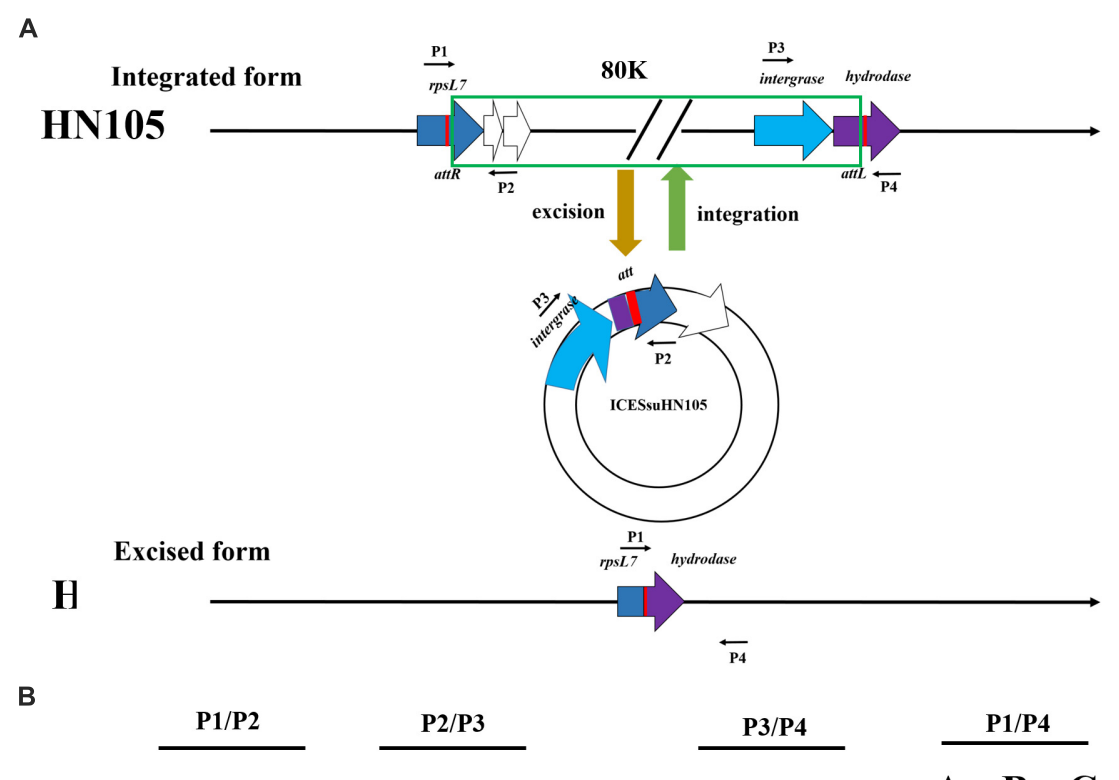

\section{$\begin{array}{lllllllllllll}\text { A } & \text { B } & \text { C } & \text { A } & \text { B } & \text { C } & \text { M } & \text { A } & \text { B } & \text { C } & \text { A } & \text { B } & \text { C }\end{array}$}

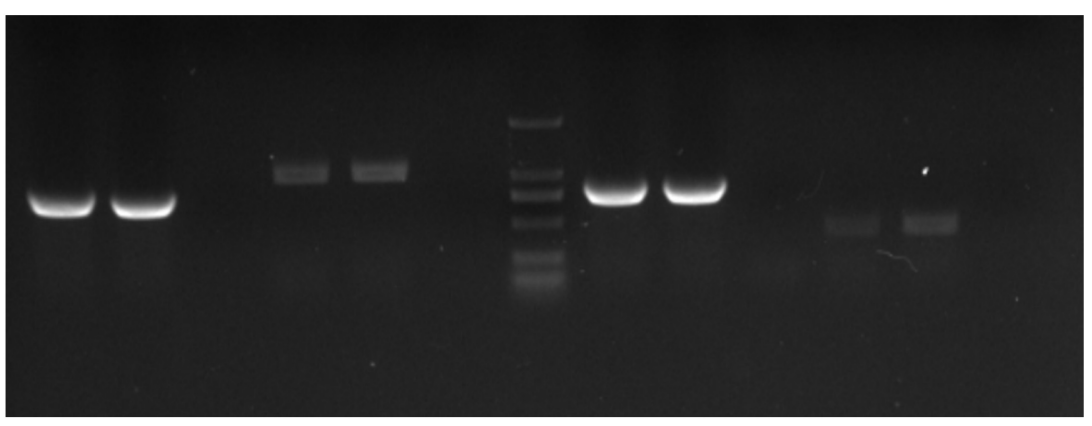

\section{A: HN105 \\ B: ZY05719 \\ C: P1/7}

M: 2000bp

FIGURE 5 | Circular form of integrative and conjugative element ICESsuHN015. (A) Diagrammatic representation of the integration and excision of ICESsuHN105 and the locations of detecting primers (shown by thin arrows) for the extrachromosomal form of ICESsD9 and genome walking assays. The left and right red rectangles represent the junctions (attL and attR), which can be formed by recombination. (B) DNA gel results of the PCR verifying the presence of the circular form of ICESsuHN105 and ICESsuZY05719.

A previous study identified that bacteriocins were not secreted in a liquid medium (Vaillancourt et al., 2015). Accordingly, we purified suicin 105 after growth on THB plates using cationic exchange and reversed-phase MS, and a bacteriocin enrichment fraction (Thiede et al., 2014). Mass spectrometry identified the type of polypeptide that shared $92.3 \%$ identity with the predicted mature suicin peptide (Supplementary Table S4). This finding confirmed the secretion of suicin 105 in HN105.

\section{DISCUSSION}

Although no S. suis SS5 outbreak or epidemic has yet been reported, SS5 has been identified as a zoonotic pathogen (Kerdsin et al., 2011; Gustavsson and Rasmussen, 2014). In this study, the isolate HN105 was considered to be non-serotypeable with the reference antisera due to auto-agglutination. More and more isolates belong are non-serotypeable according to agglutination testing in clinic (Qiu et al., 2016; Zheng et al., 2017), which causes a problem for epidemiological investigation and genetic analysis. In this way, genotyping based on molecular techniques has been proposed based on serotype-specific $w z y / w z x$ genes (Liu et al., 2013; Ishida et al., 2014; Okura et al., 2014; Athey et al., 2016), which may be a compromise to describe serotype in the genotype level. Some reports suggested that part of the non-serotypeable strains could be typed as the known serotypes by multiplex PCR, but could not be typed by the agglutination test (Zheng et al., 2015, 2017; Qiu et al., 2016). The reasons for phenotype of auto-agglutination, poly-agglutination or non-agglutination in strains with the reference antisera remain unclear until now (Zheng et al., 2015). Referring to the above studies, isolate HN105 could be grouped into serotype 5 according to capsular gene typing system. Here, the SS5 (genotype) strain HN105 presented a high virulence phenotype, similar to the representative virulent SS2 strains, in mouse and zebrafish infection models. A recent study identified a large number of virulence-related genes in S. suis that contribute to pathogenicity (Fittipaldi et al., 2012). 
A

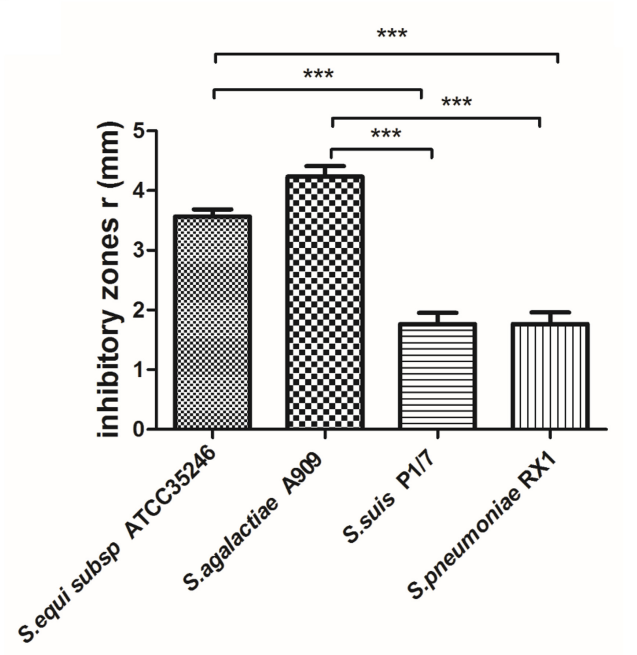

B

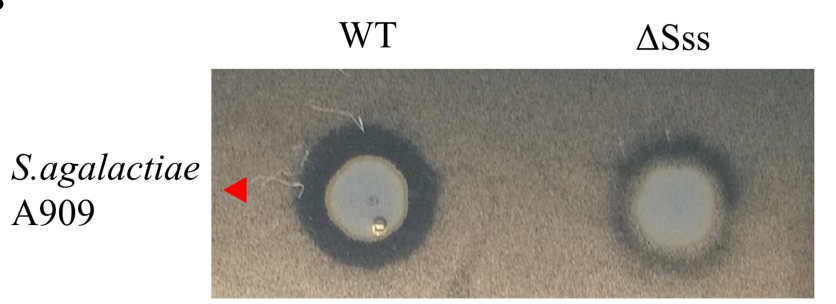

S.suis

$\Delta \mathrm{SsS}$

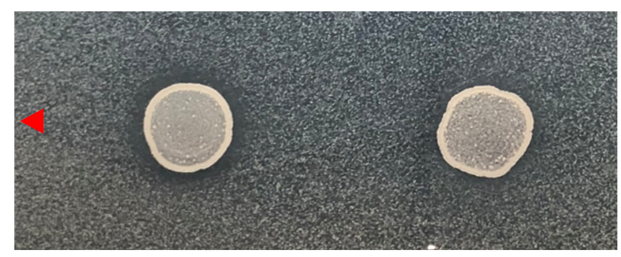

FIGURE 6 | (A) Inhibitory zones produced by S. suis HN105 against 4 Streptococcus strains. (B) Inhibitory zones produced by S. suis HN105 and mutant ( $\Delta$ Sss) against $S$. agalactiae $A 909$ and $S$. suis $\Delta$ Sss. $P<0.001$ is labeled with $* * *$

BLAST analysis of the HN105 ORFs with the reference VFs identified $75.86 \%$ of the important virulence-related genes (23/31) (Supplementary Table S3). Despite the absence of several classical virulence-associated factors such as MRP, suilysin (Sly), $\mathrm{EPF}$, and the orphan regulator RevS in HN105, some important VFs such as sortase A, dipeptidyl peptidase IV, Quorum sensing (LuxS), and other regulators (CiaRH, CovR, CcpA, and Rgglike) are still conserved in the HN105 genome. For example, cсpA encodes an important global regulator and its deletion resulted in 259 differentially expressed genes as well as a markedly reduced capsule thickness that has a significant effect on virulence (Willenborg et al., 2011; Tang et al., 2012). Sortase A controls the capacity to adhere to and invade porcine brain microvascular endothelial cells (Vanier et al., 2008). In addition, some new VFs encoding putative transcriptional regulator or flagellar protein FliS were identified in the SS5 chromosome. Moreover, we also identified a response regulator LytR, a predicted plasmid-encoded virulence factor regulator (Virulence Factors of Pathogenic Bacteria database). Such examples emphasize the genomic diversity between different serotypes in S. suis (Dong et al., 2017; Zheng et al., 2018), which highlights the value of genomic information of non-SS2 strains. The mechanisms of virulence for the HN105 strain also need to be further investigated, such as the roles of ICESsuHN105, the $43 \mathrm{~K}$ GI and $40 \mathrm{~K} \mathrm{GI}$, and whether they contribute to animal infections in China, similar to ICESsuZY05719, the well-known $89 \mathrm{~K}$ PAI, which remains unclear. We managed to test the contribution of this ICESsuHN105 in bacterial virulence with animal infection models, but it seems this 80K ICE was not required for the full virulence on HN105 isolate (Supplementary Figure S3). Thus, the following work attempts to explore its potential function on antibiotic resistance. Although $80 \mathrm{~K}$ (ICE) did not influence virulence, the $40 \mathrm{~K}$ and
$43 \mathrm{~K}$ GIs may play potential roles in the pathogenic process. In $43 \mathrm{~K}$, glycosyl transferase proteins and a Sec system were encoded. In $40 \mathrm{~K}$, a prophage was identified, which harbored some specific sections capable of opportunistic pathogenicity, including the proteolytic subunit ClpP, transcriptional regulator, holin and many hypothetical proteins. The similar structure of $50 \mathrm{~K}$ and $58 \mathrm{~K}$ GIs in SS serotype Chz strain CZ130302 (Zhang et al., 2018) had been determined play a key role in the pathogenic process. It indicated the role of these two GIs in the virulence of HN105.

Due to the widespread use of antibiotics in the porcine cultivation industry, $S$. suis is becoming increasingly resistant to numerous antibiotics (39). In this study, a novel ICESa2603 family member, ICESsuHN105, was identified in the pathogen SS5 (genotype) HN105. The strain was resistant to multiple antibiotics. Genome analysis revealed six antibiotic resistance genes in the HN105 genome that may confer resistance to diverse antibiotics by different protective pathways. For example, tet $(M)$ and tet $(O)$ were ribosomal protection proteins conferring tetracycline resistance. Specifically, the gene encodes aminoglycoside 3 '-phosphotransferase (APH), which could catalyze the transfer of the gamma-phosphoryl group from ATP to aminoglycoside antibiotics such as kanamycin, streptomycin, neomycin and lividomycin (McKay et al., 1996). Phosphorylation of the aminoglycoside antibiotics results in their inactivation in a higher survival rate of bacterial cells (Yoon et al., 2014). BLAST analysis of this gene identified homologs in known multidrug resistant pathogens such as Enterococcus faecalis, Staphylococcus aureus, and Campylobacter jejuni. HN105 might also have acquired the aph gene through horizontal gene transfer because this gene is frequently found on transposons and plasmids, and is thought to have originated as a self-defense mechanism used by the microorganisms (Shi et al., 2013). 
The similarity in the sequence identity of the integrase protein showed $>99 \%$ homology between ICESa2603 and ICESsuHN105. Therefore, we grouped ICESsuHN105 into the ICESa2603 family. Indeed, the diversity of the phylogenetic tree patterns for the backbone genes of these ICEs indicated that frequent recombination events occurred in the process of ICE formation and evolution. These recombination events may cause ICESsuHN105 to adapt to the bacterial host and specific environments. Typically, the complete ICEs are thought to be capable of mobility and can propagate to other loci upon proper stimuli.

Interestingly, a complete suicin lantibiotic locus was encoded by ICESsuHN105. The bacteriocin production system in bacteria confers a growth advantage in the interbacterial antagonism. Numerous lantibiotics have been reported in S. suis, including suicin 90-1330, suicin 3908 and suicin 65 (LeBel et al., 2014; Vaillancourt et al., 2015). Suicin 105 shares $100 \%$ homology with suicin 65. This mature lantibiotic peptide has a globular portion in the C-terminal and a linear portion in the $\mathrm{N}$-terminal. The mature suicin 105 also showed a high homology with nisin $\mathrm{H} 70$ (100\%) and streptococcin A-FF22 (84.6\%). The bacteriocin produced by these SS2 strains belonged to sequence type 28 (ST28), which is avirulent in animal models and can be treated as candidate vaccines (Athey et al., 2015). In contrast, the nisin locus found in a virulent SS5(genotype) strain HN105 genomic background could immunize and protect itself from bacteriocin killing, which may lead to failure for lantibiotic bacteriocin with potential prophylactic applications (Bierbaum and Sahl, 2009). One can easily envision a scenario where the mobile ICE with bacteriocin could integrate into other microorganisms, including other pathogens that we want to target. Such a multidrug-resistant pathogenic strain with remarkable competitive advantage for interbacterial antagonism in the environment could be a greater risk for human health.

Despite the failed in vitro conjugation assay, our DNA hybridization experiment by natural transformation resulted in the transfer of antibiotic resistance genes into different isolated genomic backgrounds. The capability of natural transformation is an important mechanism for evolution and chromosome diversity in Streptococcus species (Antunes et al., 2010). The active ComRS competence system found in the $S$. suis genome provides this strain with the ability to non-specifically uptake foreign genetic material, including genes coding for antibiotic resistance and virulence factors.

\section{CONCLUSION}

The data indicates that the SS5 (genotype) strain HN105 may be a potential source for the spread of antibiotic resistance genes and may cause serious problems regarding drug resistance.

\section{ETHICS STATEMENT}

The $\mathrm{BABL} / \mathrm{c}$ mice were purchased from the Comparative Medicine Center of Yangzhou University. Zebrafish were purchased from the Pearl River Fishery Research Institute, Chinese Academy of Fishery Science. Animal experiments were performed in the Laboratory Animal Center of Nanjing Agricultural University with the approval of the Laboratory Animal Monitoring Committee of Jiangsu Province, China (Permit number: SYXK (Su) 2017-0007). All animal experiments complied with the guidelines of the Animal Welfare Council of China.

\section{DATA AVAILABILITY}

The datasets generated for this study can be found in NCBI, The access number of the complete genome sequence for strain HN105 is CP029398.

\section{AUTHOR CONTRIBUTIONS}

YCZ, YZ, and WD conceived and designed the experiments. YCZ performed the experiments and analyzed the data. YCZ, WD, and $\mathrm{XZ}$ prepared the draft paper. ZP and JM supervised the laboratory work. JM, ZP, and HY reviewed and edited the manuscript. All authors approved the final manuscript.

\section{FUNDING}

This work was supported by the Special Fund for Public Welfare Industry of the Chinese Ministry of Agriculture (No. 201303041), the Shanghai Agriculture Applied Technology Development Program (No. G2016060201), and the project funded by the Priority Academic Program Development of Jiangsu Higher Education Institutions (PAPD). The Development Program of Jiangsu Modern Agricultural Industry Technology System Number: JSTS[2018]011.

\section{ACKNOWLEDGMENTS}

We thank all the staff for their suggestions during this research, and we would like to thank the editor for the helpful comments on this manuscript.

\section{SUPPLEMENTARY MATERIAL}

The Supplementary Material for this article can be found online at: https://www.frontiersin.org/articles/10.3389/fmicb. 2019.00274/full\#supplementary-material 


\section{REFERENCES}

Antunes, L. C., Ferreira, R. B., Buckner, M. M., and Finlay, B. B. (2010). Quorum sensing in bacterial virulence. Microbiology 156, 2271-2282. doi: 10.1099/mic.0. 038794-0

Athey, T. B., Auger, J. P., Teatero, S., Dumesnil, A., Takamatsu, D., Wasserscheid, J., et al. (2015). Complex population structure and virulence differences among serotype 2 Streptococcus suis strains belonging to sequence type 28. PLoS One 10:e0137760. doi: 10.1371/journal.pone.017 3760

Athey, T. B., Teatero, S., Lacouture, S., Takamatsu, D., Gottschalk, M., and Fittipaldi, N. (2016). Determining Streptococcus suis serotype from short-read whole-genome sequencing data. BMC Microbiol. 16:162. doi: 10.1186/s12866016-0782-8

Aziz, R. K., Bartels, D., Best, A. A., DeJongh, M., Disz, T., Edwards, R. A., et al. (2008). The RAST server: Rapid annotations using subsystems technology. BMC Genomics 9:75. doi: 10.1186/1471-2164-9-75

Bierbaum, G., and Sahl, H. G. (2009). Lantibiotics: mode of action, biosynthesis and bioengineering. Curr. Pharm. Biotechnol. 10, 2-18. doi: 10.2174/ 138920109787048616

Bland, C., Ramsey, T. L., Sabree, F., Lowe, M., Brown, K., Kyrpides, N. C., et al. (2007). CRISPR recognition tool (CRT): a tool for automatic detection of clustered regularly interspaced palindromic repeats. BMC Bioinformatics 8:209. doi: 10.1186/1471-2105-8-209

Darling, A. E., Mau, B., and Perna, N. T. (2010). progressiveMauve: multiple genome alignment with gene gain, loss and rearrangement. PLoS One 5:e11147. doi: 10.1371 /journal.pone.0011147

Delcher, A. L., Harmon, D., Kasif, S., White, O., and Salzberg, S. L. (1999). Improved microbial gene identification with GLIMMER. Nucleic Acids Res. 27, 4636-4641. doi: 10.1093/nar/27.23.4636

Dong, W., Ma, J., Zhu, Y., Zhu, J., Yuan, L., Wang, Y., et al. (2015). Virulence genotyping and population analysis of Streptococcus suis serotype 2 isolates from China. Infect. Genet. Evol. 36, 483-489. doi: 10.1016/j.meegid.2015.08.021

Dong, W., Zhu, Y., Ma, Y., Ma, J., Zhang, Y., Yuan, L., et al. (2017). Multilocus sequence typing and virulence genotyping of Streptococcus suis serotype 9 isolates revealed high genetic and virulence diversity. FEMS Microbiol. Lett. 364:fnx192. doi: 10.1093/femsle/fnx192

Fittipaldi, N., Segura, M., Grenier, D., and Gottschalk, M. (2012). Virulence factors involved in the pathogenesis of the infection caused by the swine pathogen and zoonotic agent Streptococcus suis. Fut. Microbiol. 7, 259-279. doi: 10.2217/fmb. 11.149

Fulde, M., and Valentin-Weigand, P. (2013). Epidemiology and pathogenicity of zoonotic streptococci. Curr. Top. Microbiol. Immunol. 368, 49-81. doi: 10.1007/ 82_2012_277

Gomez, E., Kennedy, C. C., Gottschalk, M., Cunningham, S. A., Patel, R., and Virk, A. (2014). Streptococcus suis-related prosthetic joint infection and streptococcal toxic shock-like syndrome in a pig farmer in the United States. J. Clin. Microbiol. 52, 2254-2258. doi: 10.1128/JCM.02934-13

Gottschalk, M., Xu, J. G., Calzas, C., and Segura, M. (2010). Streptococcus suis: a new emerging or an old neglected zoonotic pathogen? Fut. Microbiol. 5, 371-391. doi: $10.2217 / \mathrm{fmb} .10 .2$

Gustavsson, C., and Rasmussen, M. (2014). Septic arthritis caused by Streptococcus suis serotype 5 in pig farmer. Emerg. Infect. Dis. 20, 489-490. doi: 10.3201/ eid2003.130535

Holden, M. T., Heather, Z., Paillot, R., Steward, K. F., Webb, K., Ainslie, F., et al. (2009). Genomic evidence for the evolution of Streptococcus equi: host restriction, increased virulence, and genetic exchange with human pathogens. PLoS Pathog. 5:e1000346. doi: 10.1371/journal.ppat.10 00346

Huang, J., Liang, Y., Guo, D., Shang, K., Ge, L., Kashif, J., et al. (2016). Comparative genomic analysis of the ICESa2603 family ICEs and spread of erm(B)- and tet(O)-carrying transferable $89 \mathrm{~K}$-subtype ICEs in swine and bovine isolates in China. Front. Microbiol. 7:55. doi: 10.3389/fmicb.2016.00055

Huang, K., Song, Y., Zhang, Q., Zhang, A., and Jin, M. (2016). Characterisation of a novel integrative and conjugative element ICESsD9 carrying erm(B) and tet $(\mathrm{O})$ resistance determinants in Streptococcus suis, and the distribution of ICESsD9-like elements in clinical isolates. J. Glob. Antimicrob. Resist. 7, 13-18. doi: 10.1016/j.jgar.2016.05.008
Huong, V. T., Ha, N., Huy, N. T., Horby, P., Nghia, H. D., Thiem, V. D., et al. (2014). Epidemiology, clinical manifestations, and outcomes of Streptococcus suis infection in humans. Emerg. Infect. Dis. 20, 1105-1114. doi: 10.3201/ eid2007.131594

Ishida, S., Tien, L. H. T., Osawa, R., Tohya, M., Nomoto, R., Kawamura, Y., et al. (2014). Development of an appropriate PCR system for the reclassification of Streptococcus suis. J. Microbiol. Methods 107, 66-70. doi: 10.1016/j.mimet.2014. 09.003

Kerdsin, A., Dejsirilert, S., Sawanpanyalert, P., Boonnark, A., Noithachang, W., Sriyakum, D., et al. (2011). Sepsis and spontaneous bacterial peritonitis in Thailand. Lancet 378:960. doi: 10.1016/S0140-6736(11)60923-9

Kerdsin, A., Hatrongjit, R., Gottschalk, M., Takeuchi, D., Hamada, S., Akeda, Y., et al. (2017). Emergence of Streptococcus suis serotype 9 infection in humans. J. Microbiol. Immunol. Infect. 50, 545-546. doi: 10.1016/j.jmii.2015.06.011

Lagesen, K., Hallin, P., Rodland, E. A., Staerfeldt, H. H., Rognes, T., and Ussery, D. W. (2007). RNAmmer: consistent and rapid annotation of ribosomal RNA genes. Nucleic Acids Res. 35, 3100-3108. doi: 10.1093/nar/gkm160

LeBel, G., Vaillancourt, K., Frenette, M., Gottschalk, M., and Grenier, D. (2014). Suicin 90-1330 from a nonvirulent strain of Streptococcus suis: a nisin-related lantibiotic active on gram-positive swine pathogens. Appl. Environ. Microbiol. 80, 5484-5492. doi: 10.1128/AEM.01055-14

Li, M., Shen, X., Yan, J., Han, H., Zheng, B., Liu, D., et al. (2011). GI-type T4SS-mediated horizontal transfer of the $89 \mathrm{~K}$ pathogenicity island in epidemic Streptococcus suis serotype 2. Mol. Microbiol. 79, 1670-1683. doi: 10.1111/j. 1365-2958.2011.07553.x

Liu, Z., Zheng, H., Gottschalk, M., Bai, X., Lan, R., Ji, S., et al. (2013). Development of multiplex PCR assays for the identification of the 33 serotypes of Streptococcus suis. PLoS One 8:e72070. doi: 10.1371/journal.pone.0072070

Lowe, T. M., and Eddy, S. R. (1997). tRNAscan-SE: a program for improved detection of transfer RNA genes in genomic sequence. Nucleic Acids Res. 25, 955-964. doi: 10.1093/nar/25.5.955

Maiden, M. C., Jansen Van Rensburg, M. J., Bray, J. E., Earle, S. G., Ford, S. A., Jolley, K. A., et al. (2013). MLST revisited: the gene-by-gene approach to bacterial genomics. Nat. Rev. Microbiol. 11, 728-736. doi: 10.1038/nrmicro3093

McKay, G. A., Roestamadji, J., Mobashery, S., and Wright, G. D. (1996). Recognition of aminoglycoside antibiotics by enterococcal-staphylococcal aminoglycoside $3^{\prime}$-phosphotransferase type IIIa: role of substrate amino groups. Antimicrob. Agents Chemother. 40, 2648-2650. doi: 10.1128/AAC.40.11.2648

Okura, M., Lachance, C., Osaki, M., Sekizaki, T., Maruyama, F., Nozawa, T., et al. (2014). Development of a two-step multiplex PCR assay for typing of capsular polysaccharide synthesis gene clusters of Streptococcus suis. J. Clin. Microbiol. 52, 1714-1719. doi: 10.1128/JCM.03411-13

Pan, Z., Liu, J., Zhang, Y., Chen, S., Ma, J., Dong, W., et al. (2019). A novel integrative conjugative element mediates transfer of multi-drug resistance between Streptococcus suis strains of different serotypes. Vet. Microbiol. 229, 110-116. doi: 10.1016/j.vetmic.2018.11.028

Qiu, X., Bai, X., Lan, R., Zheng, H., and Xu, J. (2016). Novel capsular polysaccharide loci and new diagnostic tools for high-throughput capsular gene typing in Streptococcus suis. Appl. Environ. Microbiol. 82, 7102-7112. doi: 10.1128/AEM. 02102-16

Shi, K., Caldwell, S. J., Fong, D. H., and Berghuis, A. M. (2013). Prospects for circumventing aminoglycoside kinase mediated antibiotic resistance. Front. Cell Infect. Microbiol. 3:22. doi: 10.3389/fcimb.2013.00022

Soares, T. C., Paes, A. C., Megid, J., Ribolla, P. E., Paduan Kdos, S., and Gottschalk, M. (2014). Antimicrobial susceptibility of Streptococcus suis isolated from clinically healthy swine in Brazil. Can. J. Vet. Res. 78, 145-149.

Tang, J., Wang, C., Feng, Y., Yang, W., Song, H., Chen, Z., et al. (2006). Streptococcal toxic shock syndrome caused by Streptococcus suis serotype 2. PLoS Med. 3:e151

Tang, Y., Wu, W., Zhang, X., Lu, Z., Chen, J., and Fang, W. (2012). Catabolite control protein A of Streptococcus suis type 2 contributes to sugar metabolism and virulence. J. Microbiol. 50, 994-1002. doi: 10.1007/s12275-012-2035-3

Thiede, B., Koehler, C. J., Strozynski, M., Treumann, A., Stein, R., Zimny-Arndt, U., et al. (2014). High resolution quantitative proteomics of HeLa cells protein species using stable isotope labeling with amino acids in cell culture (SILAC), two-dimensional gel electrophoresis (2DE) and nano-liquid chromatography coupled to an LTQ-Orbitrap mass spectrometer (vol 12, pg 529, 2012). Mol. Cell. Prot. 13, 1900-1900. doi: 10.1074/mcp.A112.019372 
Vaillancourt, K., Lebel, G., Frenette, M., Fittipaldi, N., Gottschalk, M., and Grenier, D. (2015). Purification and characterization of suicin 65, a novel class I type B Lantibiotic produced by Streptococcus suis. PLoS One 10:e145854. doi: 10.1371/journal.pone.0145854

Vanier, G., Sekizaki, T., Dominguez-Punaro, M. C., Esgleas, M., Osaki, M., Takamatsu, D., et al. (2008). Disruption of srtA gene in Streptococcus suis results in decreased interactions with endothelial cells and extracellular matrix proteins. Vet. Microbiol. 127, 417-424. doi: 10.1016/j.vetmic.2007.08.032

Willenborg, J., Fulde, M., De Greeff, A., Rohde, M., Smith, H. E., ValentinWeigand, P., et al. (2011). Role of glucose and CcpA in capsule expression and virulence of Streptococcus suis. Microbiology 157, 1823-1833. doi: 10.1099/mic. 0.046417-0

Wu, Z., Wang, W., Tang, M., Shao, J., Dai, C., Zhang, W., et al. (2014a). Comparative genomic analysis shows that Streptococcus suis meningitis isolate SC070731 contains a unique 105K genomic island. Gene 535, 156-164. doi: 10.1016/j.gene.2013.11.044

Wu, Z., Wu, C., Shao, J., Zhu, Z., Wang, W., Zhang, W., et al. (2014b). The Streptococcus suis transcriptional landscape reveals adaptation mechanisms in pig blood and cerebrospinal fluid. RNA 20, 882-898. doi: 10.1261/rna.041822.113

Yoon, E. J., Goussard, S., Touchon, M., Krizova, L., Cerqueira, G., Murphy, C., et al. (2014). Origin in Acinetobacter guillouiae and dissemination of the aminoglycoside-modifying enzyme Aph(3')-VI. mBio 5:e1972-14. doi: 10.1128/ mBio.01972-14

Yoshida, H., Wada, T., Taniyama, D., and Takahashi, T. (2017). Draft Genome sequence of clinical strain TANI1 of Streptococcus suis serotype 5 isolated from a bacteremia patient in Japan. Genome Announc. 5:e260-17. doi: 10.1128/ genomeA.00260- 17

Yu, H., Jing, H., Chen, Z., Zheng, H., Zhu, X., Wang, H., et al. (2006). Human Streptococcus suis outbreak, Sichuan, China. Emerg. Infect. Dis. 12, 914-920. doi: 10.3201/eid1206.051194

Zaccaria, E., Van Baarlen, P., De Greeff, A., Morrison, D. A., Smith, H., and Wells, J. M. (2014). Control of competence for DNA transformation in Streptococcus suis by genetically transferable pherotypes. PLoS One 9:e99394. doi: 10.1371/ journal.pone.0099394

Zhang, W., and Lu, C. P. (2007). Immunoproteomics of extracellular proteins of Chinese virulent strains of Streptococcus suis type 2. Proteomics 7, 4468-4476. doi: 10.1002/pmic.200700294

Zhang, Y., Lu, P., Pan, Z., Zhu, Y., Ma, J., Zhong, X., et al. (2018). Sssp1, a Streptococcus suis fimbriae-like protein transported by SecY2/A2 system contributes to bacterial virulence. Appl. Environ. Microbiol. doi: 10.1128/AEM.01385-18 doi: 10.1128/AEM.01385-18 [Epub ahead of print].

Zheng, H., Du, P., Qiu, X., Kerdsin, A., Roy, D., Bai, X., et al. (2018). Genomic comparisons of Streptococcus suis serotype 9 strains recovered from diseased pigs in Spain and Canada. Vet. Res. 49:1. doi: 10.1186/s13567-01 7-0498-2

Zheng, H., Ji, S., Liu, Z., Lan, R., Huang, Y., Bai, X., et al. (2015). Eight novel capsular polysaccharide synthesis gene loci identified in nontypeable Streptococcus suis isolates. Appl. Environ. Microbiol. 81, 4111-4119. doi: 10. 1128/AEM.00315-15

Zheng, H., Qiu, X., Roy, D., Segura, M., Du, P., Xu, J., et al. (2017). Genotyping and investigating capsular polysaccharide synthesis gene loci of non-serotypeable Streptococcus suis isolated from diseased pigs in Canada. Vet. Res. 48:10. doi: 10.1186/s13567-017-0417-6

Conflict of Interest Statement: The authors declare that the research was conducted in the absence of any commercial or financial relationships that could be construed as a potential conflict of interest.

Copyright (c) 2019 Zhu, Zhang, Ma, Dong, Zhong, Pan and Yao. This is an open-access article distributed under the terms of the Creative Commons Attribution License (CC BY). The use, distribution or reproduction in other forums is permitted, provided the original author(s) and the copyright owner(s) are credited and that the original publication in this journal is cited, in accordance with accepted academic practice. No use, distribution or reproduction is permitted which does not comply with these terms. 